\title{
Evaluation of microlens distributions in gravitationally lensed systems based on accurate radio observations
}

\author{
Alexander F. Zakharov,${ }^{1,2,3} \dagger$ Saša Simić, ${ }^{4}$ Luka Č. Popović, ${ }^{5}$ and \\ Predrag Jovanović ${ }^{4}$ \\ ${ }^{1}$ Institute of Theoretical and Experimental Physics, Moscow, 117259, Russia \\ ${ }^{2}$ Bogoliubov Laboratory of Theoretical Physics, JINR, Dubna, Russia \\ ${ }^{3}$ CREST and NASA Research Centers, North Carolina Central University, Durham, NC \\ 27707, USA \\ ${ }^{4}$ Faculty of Science, University of Kragujevac, 34000 Kragujevac, Serbia \\ ${ }^{5}$ Astronomical Observatory, Volgina 7, Belgrade, 11160, Serbia
}

\begin{abstract}
Enormous progress is being made in developing observational facilities. As a result, there are new opportunities to observe structures at sub-mas resolution. To explore gravitationally lensed systems, we simulate radio-lobe images distorted by microlensing. We show that the positions of 'holes' in lensed images may indicate the positions of microlens groups or overdensities.
\end{abstract}

Keywords. gravitational lensing

Observational facilities continue to improve significantly, providing ever more detailed images of astronomical objects. At radio and millimeter wavelengths, it is already possible to resolve image structures as small as a few $\mu$ as; in the future this will also apply to the X-ray and infrared bands (Cash et al. 2000; Doeleman et al. 2008; Gillessen et al. 2010; Kardashev et al. 2012). Therefore, detailed object structures will be accessible for inspection and analysis. The RadioAstron ground-space interferometer is currently operational. Here, we discuss opportunities to derive information about the distribution of microlenses in the gravitational-lens context using these facilities.

We consider gravitationally lensed systems and analyze radio-lobe structures of lensed object images. We discuss one such case, where microlenses produce observable 'holes' in the image. These structures are generated by the action of microlenses in a galaxy in the form of individual stars or groups of stars, fragmented into stellar clusters in galactic halos. We modeled one such case using a ray-shooting method to obtain the magnification map in the source plane. Similarly to Kayser et al. (1986), Schneider \& Weiss (1987), Wambsganss (1990, 2001), and Treyer \& Wambsganss (2004), we followed the light rays from the source elements toward the lens, and by accounting for them at any pixel, we were able to reproduce the image seen by the observer in the lens plane. We found that the images contain structural details in the form of areas with lower or no light emission, which we call holes. These structures are spatially connected with the appropriate microlens distributions in the perturbing galaxy and their size depends on the lens mass. We have completed a number of simulations to investigate the importance of some of the free parameters on the properties of the holes, particularly of the number of microlenses and the source size. The results of our calculations help us to express the basic properties of the holes and assess their applications in the context of future observations.

\section{† E-mail: zakharov@itep.ru}




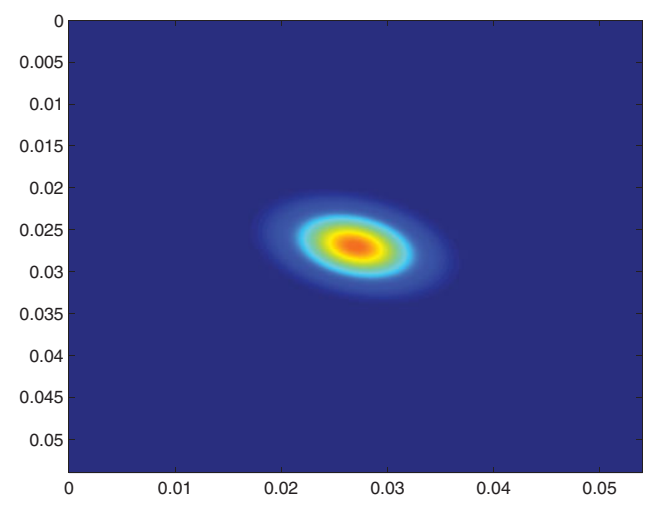

Figure 1. 2D elliptical Gaussian model adopted for the emissivity of an extended source.

In our calculations, we used a ray-shooting method (Kayser et al. 1986; Schneider \& Weiss 1987; Wambsganss 1990, 2001; Popović et al. 2006; Jovanović et al. 2008). In the framework of our approach, we simulate the microlensing magnification pattern by a random distribution of microlenses in the lens plane by shooting a beam of light rays from the observer through the lens plane towards the source. The light rays are deflected due to gravitational lensing and they reach the source plane, which contains an extended source. The magnification's spatial distribution in the source plane is proportional to the concentration of light rays at a given position.

In the square region around a source location of size $L_{\mathrm{s}}$, we introduce a grid of $N_{\mathrm{p}}^{2}$ source elements (pixels) of size $l_{\mathrm{p}}=L_{\mathrm{s}} / N_{\mathrm{p}}$. We use standard notations to define the angular-diameter distances between observer and lens, $D_{d}$, observer and source, $D_{s}$, and lens and source, $D_{d s}$ (see, e.g., Schneider et al. 1992; Peebles 1993) and define the Einstein radius as

$$
R_{\mathrm{E}}=\left(\frac{4 G M}{c^{2}} \frac{D_{s} D_{d s}}{D_{d}}\right)^{1 / 2}
$$

The lens plane is divided into small elements for which we calculate a gravitational potential, which deflects light rays. For all light rays, simulated fluxes for the corresponding image elements are proportional to the emissivity of the respective source elements. This way, we simulate image fluxes corresponding to the positions and emissivity of the source elements.

We model an extended source intensity as a 2D elliptical Gaussian function following Eq. (1). A representation of this distribution is shown in Fig. 1, where we have imposed a cutoff at around $10 \%$ of the maximum intensity $\left(0.1 I_{\max }\right)$, i.e., we use the expression

$$
g(x, y)=I_{\max } \exp \left(-\left(a\left(x-x_{0}\right)^{2}+2 b\left(x-x_{0}\right)\left(y-y_{0}\right)+c\left(y-y_{0}\right)^{2}\right)\right),(1)
$$

where the coefficients $(a, b, c)$ are given by

$$
\begin{aligned}
& a=\frac{\cos ^{2} \phi}{2 s_{x}^{2}}+\frac{\sin ^{2} \phi}{2 s_{y}^{2}} \\
& b=-\frac{\sin 2 \phi}{4 s_{x}^{2}}+\frac{\sin 2 \phi}{4 s_{y}^{2}} \\
& c=\frac{\sin ^{2} \phi}{2 s_{x}^{2}}+\frac{\cos ^{2} \phi}{2 s_{y}^{2}} .
\end{aligned}
$$


Here $\phi$ is angle in the $x-y$ plane, and $s_{x}$ and $s_{y}$ are the Gaussian widths. This approach allows us to adjust the size, orientation, and intensity distribution of the source.

To study the effects of microlensing on the distortion of an extended source, we performed simulations for a set of parameters. We chose a particular size in the source plane of at least $30 R_{\mathrm{E}}$, at a redshift $z_{s}=2$, and we assume that the lens is located at $z_{d}=1$.

First, we performed simulations for different numbers of microlenses comprising a lens by changing the convergence parameter $\kappa_{\star}$. Increasing the number of stars leads to the appearance of dark spots ('holes') in the simulated images (see Fig. 2).

Lens plane image

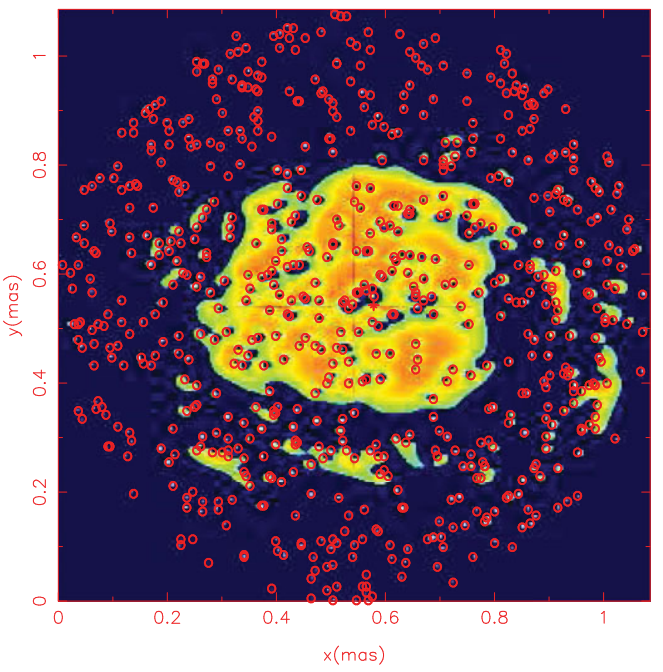

Lens plane image

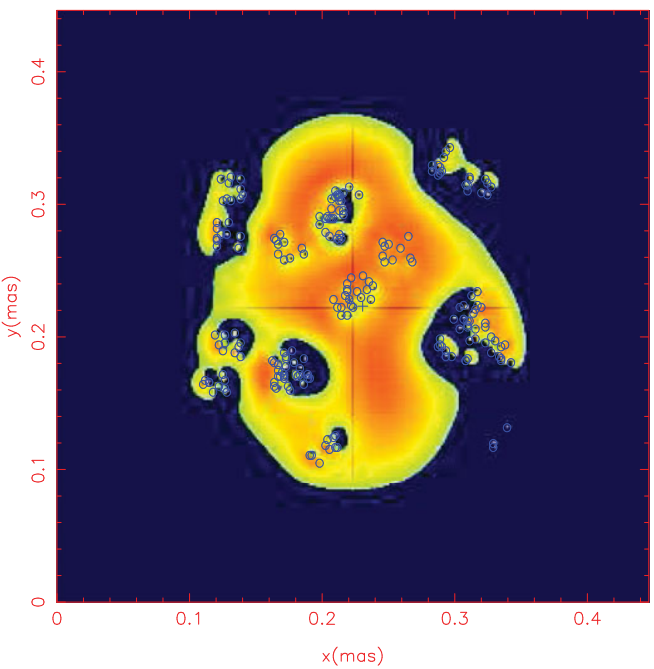

Figure 2. Two examples of distorted images and microlens distributions.

We also study the size variations of the holes caused by different locations of a small group of microlenses. It is interesting to note that the holes are located between the projection of the source center in the lens plane and the group of microlenses. As the distance between the group of microlenses and the source center's projection decreases, the hole sizes also decrease, until they disappear. Also, by examining the image intensities, one can see that the intensity increases in the vicinity of the holes in the opposite direction with respect to the source center's projection.

This effect is always present but is more strongly expressed towards the outer edge of the source. For lens positions closer to the central region, there is an apparent 'equilibrium' of light from both sides of a source, which causes the holes to decrease in diameter and almost disappear in the center. In Fig. 3 we present the dependence of the number of holes on the number of stars in the lens. There is a trend of increasing numbers of holes with increasing numbers of microlenses, but in some cases we notice slight variations. These can be explained by the effects of stellar groups, which means that a single hole can be produced by a group of stars. In Fig. 4 we examine the dependence of the hole dimensions on the source dimensions. As expected, the hole dimensions in the source image in the lens plane decrease with increasing source diameter. This could be explained by the fact that if more light rays originate from an intense and extended source, fewer of them will be successfully deflected, thus causing a reduction in the hole size.

Therefore, we claim that holes in images of gravitationally lensed systems may indicate the locations of microlens clusters as well as their sizes. 


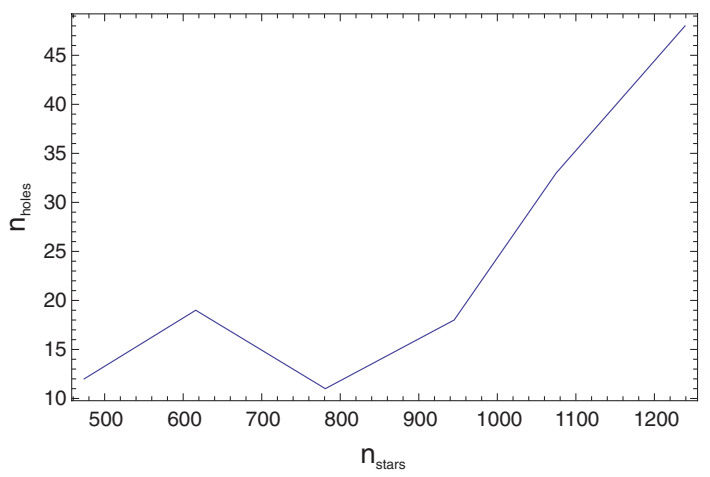

Figure 3. Dependence of the number of 'holes' on the number of lensing stars (microlenses).

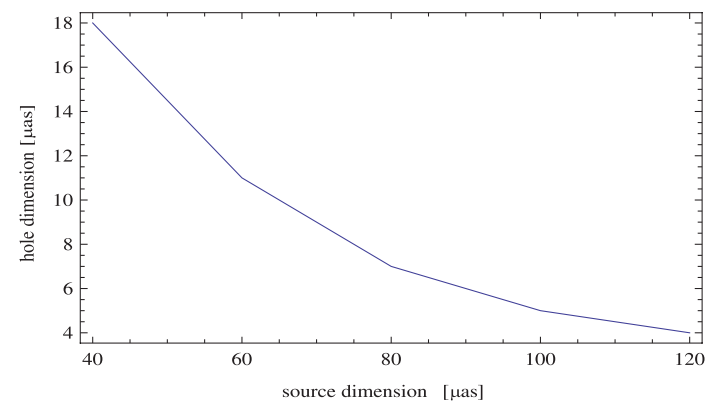

Figure 4. Dependence of the size of the 'holes' on the size of the source.

\section{Acknowledgements}

This work is a part of projects 176003, 'Gravitation and the Large Scale Structure of the Universe' and 176001, 'Astrophysical Spectroscopy of Extragalactic Objects,' supported by the Serbian Ministry of Education, Science and Technological Development. We acknowledge Richard de Grijs for his kind attention to our contribution and his careful editing of our manuscript.

\section{References}

Cash, W., Shipley, A., Osterman, S., \& Joy, M. 2000, Nature, 407, 160

Doeleman, S. S., Weintroub, J., Rogers, A. E. E., et al. 2008, Nature, 455, 78

Jovanović, P., Zakharov, A. F., Popović, L.Č., \& Petrović, T. 2008, MNRAS, 386, 397

Gillessen, S., Eisenhauer, F., Perrin, G., et al. 2010, Proc. SPIE, 7734, 77340Y

Kardashev, N. S., et al. 2012, http://www.asc.rssi.ru/radioastron/index.html

Kayser, R., Refsdal, S., \& Stabell, R. 1986, A\& A, 166, 36

Peebles, P. J. E. 1993, Principles of Physical Cosmology, Princeton Univ. Press: Princeton

Popović, L.Č., Jovanović, P., Mediavilla, E., Zakharov, A. F., Abajas, C., Munoz, J. A., \& Chartas, G., 2006, ApJ, 637, 630

Schneider, P., Ehlers, J., \& Falco, E. E. 1992, Gravitational Lenses, Springer-Verlag: Berlin

Schneider, P. \& Weiss, A. 1987, A\&SA, 171, 49

Treyer, M. \& Wambsganss, J. 2004, A\&A, 19, 416

Wambsganss, J. 1990, Ph.D. Thesis, Univ. of Munich (Report MPA 550)

Wambsganss, J. 2001, Publ. Astron. Soc. Aus., 18, 207 